\title{
Bevacizumab in Recurrent Glioblastoma: Five Informative Patient Scenarios
}

\author{
Warren P. Mason
}

\begin{abstract}
Glioblastoma is the most common and malignant primary brain tumour in adults. Maximum feasible surgical resection, radiotherapy and temozolomide chemotherapy at initial diagnosis have improved prognosis but rapid recurrence is typical and survival remains brief. There is an urgent need for effective new treatments and approval of the antiangiogenic agent bevacizumab for recurrent glioblastoma by Health Canada in 2009 has been the most notable recent therapeutic advance for this disease. This review with illustrative case studies highlights how bevacizumab has been incorporated into the treatment of glioblastoma in Canada and describes the ongoing controversies surrounding its clinical application.
\end{abstract}

RÉSUMÉ: Le bévacizumab dans le traitement de la récidive du glioblastome : cinq scenarios informatifs. Le glioblastome est la tumeur primitive du cerveau qui est la plus maligne et la plus fréquente chez les adultes. La résection chirurgicale maximale réalisable, la radiothérapie et la chimiothérapie au moyen du témozolomide au moment du diagnostic initial en ont amélioré le pronostic, mais une récidive rapide est typique et la survie demeure brève. Il est urgent de développer de nouveaux traitements efficaces. L'approbation par Santé Canada en 2009 de l'agent antiangiogénique bévacizumab pour traiter la récidive du glioblastome a été le traitement innovateur le plus notable pour cette maladie. Cette revue souligne comment le bévacizumab a été incorporé au traitement du glioblastome au Canada, avec des études de cas à l'appui, et décrit les controverses qui ont cours concernant son utilisation en clinique.

Keywords: Angiogenesis, bevacizumab, glioblastoma, malignant glioma, vascular endothelial growth factor

doi:10.1017/cjn.2015.21

Can. J. Neurol. Sci. 2015; 42: 149-156

Glioblastoma is the most common and aggressive primary brain tumour in adults with an incidence of approximately 3.1 per 100000 in the United States. Survival for patients with glioblastoma is dismal, with rates in the range of $33.7 \%$ and $4.5 \%$ at one and five years, respectively. ${ }^{1}$ Since 2005 , standard initial management for most patients with glioblastoma consists of maximal safe resection followed by external beam radiotherapy with systemic temozolomide chemotherapy. ${ }^{2}$ The incorporation of temozolomide into the therapeutic armamentarium for this disease represents the first clinical advance in decades improving median overall survival from 12.1 months to 14.6 months. However, the prognosis for most patients with glioblastoma in Canada has not been substantially altered by temozolomide, as less than $50 \%$ receive this therapy at diagnosis and, amongst those that do, only $35-45 \%$ have tumours with $\mathrm{O}^{6}$-MethylguanineDNA methyltransferase (MGMT) promotor hypermethylation, a molecular genetic predictor of favorable response to this new radiochemotherapeutic regimen. 3

The vast majority of patients with glioblastoma experience disease progression with a median time to recurrence being in the range of seven months from diagnosis. ${ }^{5}$ At progression, there is no standard of care although further surgery, palliative chemotherapy and occasionally re-irradiation are employed. Despite ongoing efforts to develop novel therapies for glioblastoma, no effective drugs have been clearly identified. At progression, the use of conventional chemotherapy or experimental anti-tumour therapies is associated with a six-month progression-free survival in the range of $10-15 \%$ and a median overall survival in the range of 25-30 weeks. ${ }^{6,7}$
Glioblastoma is a highly vascular neoplasm and the extent of microvascular proliferation correlates with aggressive behavior and poor survival. ${ }^{8}$ Vascular endothelial growth factor (VEGF)-A expression has been implicated in glioblastoma angiogenesis: increased production is correlated with tumour progression and overexpression is predictive of poor prognosis. Vascular endothelial growth factor and related signaling cascades have been identified as key therapeutic targets in glioblastoma and efforts to inhibit these pathways have driven the clinical evaluation of numerous antiangiogenic agents for this disease. ${ }^{9}$

Bevacizumab is a humanized monoclonal antibody that inhibits VEGF and has been approved for use in a number of systemic cancers. In 2009, Health Canada and the Food and Drug Administration in the United Stated granted accelerated and conditional approval of bevacizumab in the first- or second-line treatment of recurrent glioblastoma based on two noncomparator trials that evaluated this agent alone and with irinotecan. ${ }^{10}$ Both studies documented remarkable radiographic responses in the range of $28-35 \%$ and six-month progression-free survival in the range of 29-43\% despite a somewhat disappointing median overall survival of only 7.8-9.2 months. ${ }^{11,12}$ In these studies, bevacizumab was well tolerated with no unexpected treatment-related adverse events reported.

\footnotetext{
From the Department of Medicine, Princess Margaret Cancer Centre and University of Toronto, Toronto, Ontario, Canada.

Received November 27, 2014. Final Revisions Submitted January 20, 2015. Correspondence to: Warren P. Mason, Princess Margaret Cancer Centre, 610 University Avenue, Suite 18-717, Toronto, ON, M5G 2M9, Canada. Email: Warren.mason@uhn.ca
} 
In Canada, since Health Canada approval, the use of bevacizumab for recurrent glioblastoma has increased steadily to the extent that this agent has become a common therapeutic option. While patients who have apparent radiographic responses to bevacizumab typically experience improvement and even resolution of neurologic and neurocognitive deficits, reduction in corticosteroid requirements and enhanced quality of life with manageable and expected toxicities, considerable controversy surrounds the use of this drug because its impact on survival remains questionable. ${ }^{13,14}$ Additionally, ongoing issues on how best to use this agent remain unresolved. For instance, unlike systemic cancers where there appears to be synergistic benefit from the administration of bevacizumab with chemotherapy, it is not clear whether bevacizumab should be administered alone or with chemotherapy when used for recurrent glioblastoma. Moreover, the optimal timing of bevacizumab for recurrent glioblastoma remains uncertain: should it be used at first progression or at subsequent progressions? Because bevacizumab has not extended survival of patients with recurrent glioblastoma as dramatically as it has altered tumour appearance on MR scans, real concern surrounds the clinical significance of the often impressive radiographic responses associated with bevacizumab use. Finally, as with all drugs, bevacizumab is not universally and predictably effective for all patients with progressive glioblastoma. The absence of a reliable biomarker of response for bevacizumab has frustrated efforts to identify the best candidates for this expensive treatment that is not without serious and potentially life-threatening side effects. Nonetheless, bevacizumab has become a standard therapy for patients with recurrent glioblastoma in Canada and increasing clinical experience has demonstrated the potential value of this agent for a disease where effective treatments are lacking. The patient scenarios reported in this case series are illustrative of the various ways bevacizumab is being used for patients with recurrent glioblastoma and of how this drug is altering the lives of patients with this fatal and devastating illness.

\section{Case 1}

Bevacizumab used as monotherapy at first progression in a symptomatic patient who experiences a prolonged radiographic response and clinical improvement in neurologic deficits and quality of life

A 63 year-old man with controlled hypertension presented with a short history of language disturbance, incoordination of his right hand and possible partial motor seizures in June 2012. A magnetic resonance (MR) scan of the brain disclosed two enhancing lesions involving the left premotor gyrus, biopsy confirmed as glioblastoma. He commenced dexamethasone, two mg daily and levetiracetam $1000 \mathrm{mg}$ twice daily (BID). His performance status at diagnosis was Eastern Cooperative Oncology Group (ECOG) performance status 0. He received cranial radiotherapy 60 Gray (Gy) with concomitant temozolomide commencing July 2012. After completion of radiotherapy, adjuvant treatment with temozolomide was initiated in September 2012. After receiving a sixth cycle of temozolomide in February 2013, an MR scan from March 2013 disclosed tumour progression with more enhancement and worsened fluid-attenuated inversion recovery (FLAIR) abnormality. Clinically he had deteriorated with worsened seizures, increased weakness of his right hand and a performance status of ECOG 2. Dexamethasone $4 \mathrm{mg}$ BID was started and clobazam $5 \mathrm{mg}$ daily was added to his anticonvulsant regimen. During a clinic visit in March 2013, after having received one cycle bevacizumab at a dosage of $10 \mathrm{mg} / \mathrm{kg}$, he was noted to have markedly improved strength and no recent seizures. Dexamethasone was tapered and discontinued the following week. His performance status had improved to ECOG 1. An MR scan in April revealed improvement in contrast enhancing and FLAIR abnormalities. By May 2013, he had no neurologic deficits but worsening hypertension required the addition of candesartan plus to his antihypertensive regimen of enalapril and furosemide. By October 2013, he had been receiving bevacizumab for eight months and he remained radiographically stable with no neurologic deficits. However, shortly thereafter, he experienced worsened partial seizures, developed occasional word finding difficulty and had fallen once. Mild right-sided weakness was noted on examination. Clobazam was increased to $20 \mathrm{mg}$ daily and bevacizumab administration continued. He was admitted to hospital in November 2013 with intractable seizures and progressive right-sided weakness. An MR scan from this admission revealed tumour progression with new enhancing disease. Bevacizumab was discontinued after nine months administration, dexamethasone $4 \mathrm{mg}$ four times a day (QID) was initiated and he was discharged home. Later that month, when assessed in clinic, he was unable to walk, was cognitively impaired, and had a performance status of ECOG 3. At his request he was transferred to palliative care where he died 30 months after diagnosis and six weeks after discontinuing bevacizumab.

\section{Case 2}

Bevacizumab used as monotherapy in a patient with poor performance status after progression on temozolomide and third craniotomy with dramatic response

A 56 year-old woman, previously healthy, experienced a first generalized tonic clonic seizure. An MR scan of the brain revealed a right frontal lesion and a craniotomy with partial resection of a glioblastoma was performed in September 2010. Post-operatively the patient remained without fixed neurologic deficits. Between September and October 2010, she received cranial irradiation 60 Gy with concurrent temozolomide. Treatment was complicated by mild thrombocytopenia. When assessed in December 2010, the patient remained intact neurologically and an MR scan revealed interval improvement. In January 2011, a decision to defer adjuvant temozolomide was made due to persistent thrombocytopenia. By April 2011, the patient had developed a new left hemiparesis and dexamethasone was started at $4 \mathrm{mg}$ QID. Temozolomide chemotherapy was initiated for tumour progression evident on an emergent computed tomography (CT) scan of the brain. In May 2011, with a follow-up MR brain confirming tumour progression, a second cycle of temozolomide was administered and dexamethasone was reduced to $4 \mathrm{mg}$ BID because of significant improvement of hemiparesis. The patient continued to deteriorate and she elected for a second surgery with partial tumour resection later that month. Because the patient had limited temozolomide exposure and because the clinical deterioration for which surgery was performed may have been related to a reduction in dexamethasone, a third cycle of temozolomide was given in June 2011. By late September 2011, however, worsening hemiparesis required an increase in dexamethasone dose to $12 \mathrm{mg}$ daily. By October 2011, the patient was wheelchair bound and 

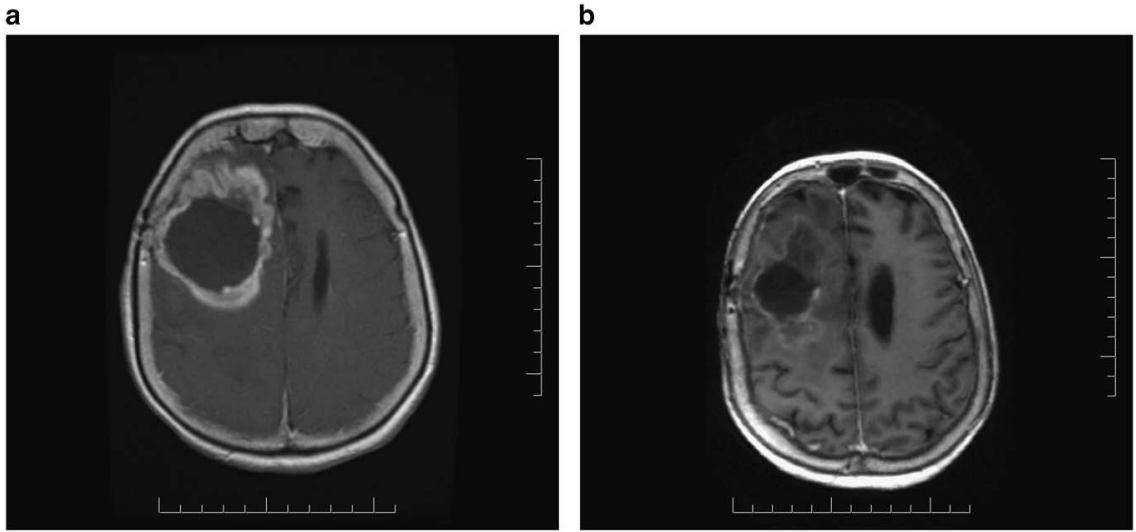

Figure 1: (a) Contrast-enhanced axial T1-weighted MR scan reveals considerable tumour progression and surrounding edema following a second resection for recurrent glioblastoma. (b) Following two infusions of bevacizumab, considerable improvement of cerebral edema and enhancing tumour is noted.

dexamethasone was increased to $24 \mathrm{mg}$ daily without benefit. Later that month, the patient underwent a third craniotomy that was complicated by a peri-operative pulmonary embolism. Post-operatively, she remained in a wheelchair with a performance status of ECOG 4. The patient commenced bevacizumab at a dosage of $10 \mathrm{mg} / \mathrm{kg}$ every two weeks after a post-operative MR scan of the brain dated November 2011 revealed further tumour growth (Figure 1a). By December 2011 she had experienced dramatic improvement in her hemiparesis and was able to walk without assistance. Her performance status improved to ECOG 2 and dexamethasone was reduced to $4 \mathrm{mg}$ BID. An MR scan in January 2012 was dramatically improved (Figure 1b). The patient continued receiving bevacizumab but by March 2012, while clinically stable, had radiographic evidence suggestive of tumour progression with worsening FLAIR abnormality. Etoposide chemotherapy was added and bevacizumab continued. Despite this change in treatment, the patient deteriorated clinically and treatment was discontinued the following month. The patient was transferred to palliative care and expired of progressive disease in July 2012 having received bevacizumab with benefit for 5 of the 22 months survival from diagnosis.

\section{Case 3}

Life-threatening complication and impaired wound healing after brief bevacizumab exposure in a patient with a recurrent glioblastoma.

A 41 year-old woman presented with a three-year history of vague headaches and syncope in November 2012. In December she underwent a craniotomy for resection of a left parietal glioblastoma. Following surgery, she remained neurologically intact with a performance status of ECOG of 0 and she subsequently received radiotherapy $60 \mathrm{~Gy}$ with concurrent temozolomide but received only one cycle of adjuvant temozolomide because of profound and persistent thrombocytopenia. In December 2013, radiographic progression was noted and temozolomide was resumed on a continuous schedule. An MR scan performed in February 2014 disclosed tumour progression and treatment with lomustine at a dosage of $80 \mathrm{mg} / \mathrm{m}^{2}$ every six weeks with bevacizumab at a dosage of $10 \mathrm{mg} / \mathrm{kg}$ every two weeks was initiated.
Following one cycle of lomustine and two cycles of bevacizumab profound myelosuppression resulted in cessation of chemotherapy and interruption of bevacizumab. In June 2014 an MR scan disclosed tumour progression; craniotomy with tumour resection was performed. In September 2104, despite stable MR imaging, the patient deteriorated clinically with the development of new expressive aphasia and a performance status of ECOG 1 and bevacizumab treatment was resumed. In October 2014, after three bevacizumab infusions, the patient presented with acute abdominal pain necessitating an emergent laparotomy and Hartman's procedure for management of a perforated sigmoid colon affected with diverticulosis. Bevacizumab therapy was discontinued and the patient was transferred to palliative care. Due to recent bevacizumab exposure she required repair of a dehisced wound in November 2014.

\section{Case 4}

Bevacizumab initiated at third progression in a patient with glioblastoma with prolonged response. Lomustine added at time of fourth progression with disease stabilization.

A 32 year-old man presented with a generalized tonic-clonic seizure in June 2008. An MR scan revealed a right frontal lobe lesion suggestive of a glioblastoma and a craniotomy with partial tumour resection confirmed this diagnosis. He was otherwise healthy and had a completely normal neurologic examination. He received radiotherapy 60 Gy with concurrent temozolomide between July and September 2008. An MR scan performed in October 2008 revealed a small enhancing nodule within a larger bed of residual nonenhancing disease. He enrolled in a clinical trial and received adjuvant temozolomide with everolimus, an mTOR inhibitor. An MR scan performed in December 2008 after two cycles of temozolomide with the mTOR inhibitor revealed disease progression. The experimental agent was discontinued and continuous daily temozolomide commenced with subsequent radiographic stabilization. He received a total nine cycles of continuous monthly temozolomide, completing treatment in October 2009. While remaining clinically asymptomatic but for occasional partial complex seizures, he resumed temozolomide chemotherapy in August 2011 due to slow progression of 


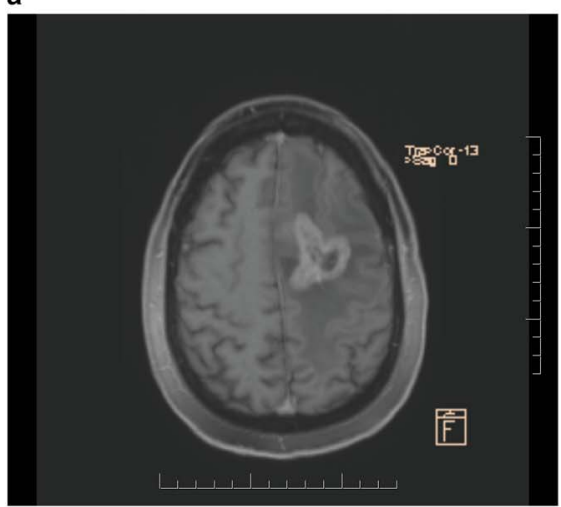

c

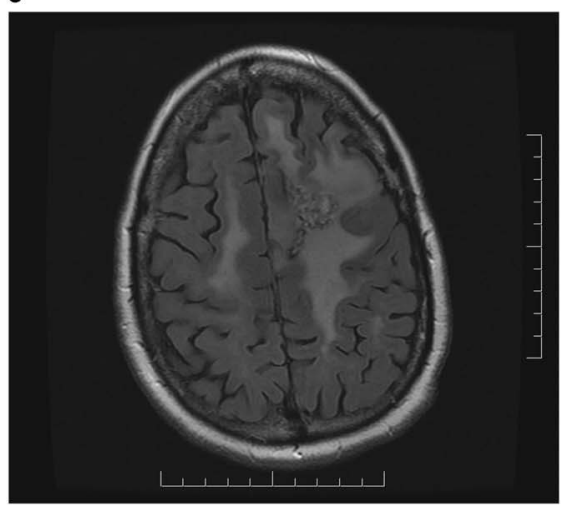

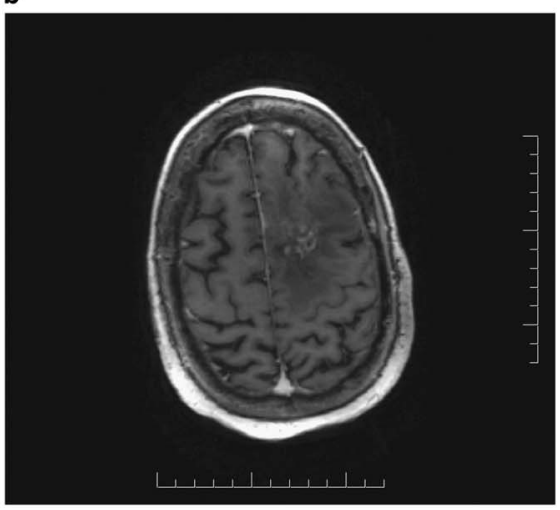

d

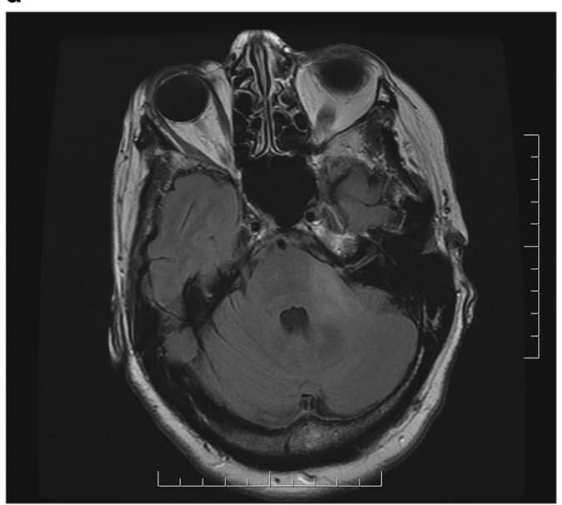

Figure 2: (a) Contrast-enhanced axial Ti-weighted MR scan demonstrates enhancing tumour and surrounding edema in left frontal lobe. (b) After one cycle of lomustine chemotherapy and two infusions of bevacizumab, significant reduction of enhancing disease and edema is apparent. (c) After 12 months of lomustine and bevacizumab treatment, axial FLAIR imaging discloses new widespread abnormalities in both cerebral hemispheres and (d) brainstem in the region of the left brachium conjunctivum consistent with the development of nonenhancing infiltrative disease progression.

nonenhancing residual disease on MR imaging. He received nine monthly cycles of continuous daily temozolomide, completing treatment in July 2012 with disease stabilization on MR imaging. When radiographic progression was noted in November 2012, a repeat craniotomy with resection of tumour was performed in December 2012. He remained well without neurologic deficits, continuing to work and with a performance status of ECOG 0. He started bevacizumab monotherapy at a dosage of $10 \mathrm{mg} / \mathrm{kg}$ every two weeks in January 2013 with marked radiographic improvement noted on MR imaging performed in March 2013. He continued receiving bevacizumab monotherapy, tolerating this treatment without incident until an MR scan performed in November 2013 revealed progressive disease in the right frontal lobe with new involvement in the left frontal lobe. Lomustine chemotherapy at a dosage of $110 \mathrm{mg} / \mathrm{m}^{2}$ every six weeks was added to bevacizumab in December 2013. An MR scan performed in March 2014 disclosed disease stabilization. He received a seventh cycle of lomustine with bevacizumab in August 2014. He remains clinically stable with occasional partial complex seizures reasonably well controlled with levetiracetam, continues to work and has an ECOG 0 performance status. Altogether he has received bevacizumab for 19 months; at progression on bevacizumab monotherapy, the addition of lomustine has resulted in disease stabilization for the past nine months.

\section{Case 5}

Bevacizumab and lomustine used together at first progression in a patient with glioblastoma with durable clinical and radiographic response

A 52 year-old woman with controlled hypertension developed seizures and mild aphasia in July 2012. An MR scan revealed a left frontal lobe lesion, initially vague, but when imaging was repeated one month later, a distinct enhancing abnormality was apparent. A craniotomy was performed in September 2012 and a diagnosis of glioblastoma was established. She received radiotherapy 60 Gy with concurrent temozolomide between September and November 2012. During irradiation, she required increasing doses of dexamethasone to $12 \mathrm{mg}$ daily and levetiracetam to 1000 BID to control worsening symptoms and seizures. Adjuvant temozolomide commenced in December 2012. After three cycles of adjuvant temozolomide, imaging was suggestive of progression with worsening of FLAIR abnormality. Daily continuous temozolomide was initiated in March 2013. An MR scan performed in May 2013 revealed progressive tumour enhancement (Figure 2a). Clinically, she was suffering from worsening aphasia and right-sided hemiparesis and uncontrolled seizures despite increasing levetiracetam to $1500 \mathrm{mg}$ BID. Dexamethasone at a dose of $8 \mathrm{mg}$ BID was prescribed. Her performance status had 
deteriorated to ECOG 3. In July 2013, lomustine chemotherapy at a dosage of $110 \mathrm{mg} / \mathrm{m}^{2}$ every six weeks was prescribed. Bevacizumab at a dosage of $10 \mathrm{mg} / \mathrm{kg}$ every two weeks commenced in August 2013 and after two infusions the patient reported improved language function and right-sided hemiparesis. Her performance status had improved to ECOG 2. An MR scan performed in September 2013 showed improvement with less enhancing abnormality (Figure 2b). A second cycle of lomustine was dispensed in October 2013. By this time she no longer required corticosteroids. An MR scan performed in November 2013 revealed further improvement. Symptoms of hemiparesis and aphasia had improved further. She experienced ongoing problems with hypertension, treated effectively with ramapril and amlodipine. After five cycles of lomustine with biweekly bevacizumab an MR scan in March 2014 disclosed stable enhancing disease but slightly increased FLAIR abnormality. In July 2014, after eight cycles of lomustine with bevacizumab, treatment was suspended due to thrombocytopenia (platelets 64). She had received lomustine and bevacizumab for 12 months with benefit and manageable toxicities, but with tolerance for further chemotherapy was now compromised, only bevacizumab was resumed after thrombocytopenia had improved. However, she rapidly deteriorated clinically with worsening right-sided hemiparesis and an MR scan in August 2014 revealed evidence of extensive non-enhancing disease progression in both cerebral hemispheres and the brainstem in the region of the left brachium conjunctivum (Figures $2 \mathrm{c}$ and d). Bevacizumab was discontinued and the patient was transferred to palliative care.

\section{DisCUSSION}

Since 2009, bevacizumab has increasingly been incorporated in the management of patients with glioblastoma, despite persistent concerns surrounding the survival value of this agent for recurrent glioblastoma. Radiographic responses, on average in the range of three to six months, are often transient and the median overall survival of eight to nine months reported by landmark trials of bevacizumab regimens for recurrent glioblastoma suggest a very modest improvement in survival, perhaps just two to three months, although a survival benefit has not been proven. ${ }^{11,12}$ Nonetheless, the use of bevacizumab in progressive glioblastoma has become widespread and is now entrenched in clinical practice to the extent where population-based studies are detecting its impact on survival. An analysis of the Surveillance, Epidemiology, and End Results (SEER) registry in the United States suggests that, following Food and Drug Administration approval of bevacizumab for recurrent glioblastoma in 2009 in the United States, survival for patients with glioblastoma improved. ${ }^{15}$ In this analysis, patients with glioblastoma who died in 2010 with presumed access to bevacizumab survived a median of two months longer than those who died in 2008. This difference, albeit small, was highly statistically significant and likely underestimated a more impressive improvement of median survival of subpopulations of patients who received bevacizumab therapy. These data are the strongest to date for suggesting that bevacizumab is a useful treatment for recurrent glioblastoma, confirming the value of the radiographic response data that resulted in Food and Drug Administration and Health Canada approval of bevacizumab for recurrent glioblastoma as a good predictor of survival benefit.
The use of bevacizumab is associated with numerous toxicities, some of which can be life threatening. However, considerable published experience using bevacizumab alone and in combination with various chemotherapeutic agents has not identified unique toxicities in patients with glioblastoma. ${ }^{11,12,31,32}$ Nonetheless, while bevacizumab use in patients with glioblastoma is generally considered safe, side effects such as venous thromboembolism, bowel perforation, ischemic stroke, intra-tumoural and cerebral hemorrhage and impaired wound healing following surgical procedures such as craniotomy can complicate management of patients with glioblastoma and must be carefully considered when choosing to use this drug in this clinical setting.

The approval of bevacizumab for recurrent glioblastoma was based on studies that evaluated this agent alone and with irinotecan. Because irinotecan did not appreciably improve overall survival, there was little appeal for use of this agent in clinical practice. However, many patients do not achieve adequate responses to bevacizumab alone and, for those who do, responses are often transient. The search for an effective agent to combine with bevacizumab in the recurrent setting has prompted numerous trials of bevacizumab with chemotherapies such as temozolomide, etoposide, carboplatin and lomustine and novel targeted therapies such as erlotinib, temsirolimus and sorafenib. ${ }^{16-19}$ Of these, the only drug that demonstrates tentative signs of benefit when combined with bevacizumab is lomustine, having been evaluated by the Dutch in a randomized phase II study of bevacizumab versus bevacizumab plus lomustine versus lomustine single agent in recurrent glioblastoma (BELOB trial). ${ }^{20}$ The preliminary analysis of this study has suggested that bevacizumab and lomustine have similar activity when used alone (progression-free survival at six months of $11 \%$ versus $18 \%$, respectively, and overall survival of eight months in both arms) but generate a $41 \%$ progression-free survival at six months and an overall survival of 11 months when used together. In this trial, bevacizumab was administered at a dosage of $10 \mathrm{mg} / \mathrm{kg}$ every two weeks and lomustine was administered at a dosage of $110 \mathrm{mg} / \mathrm{m}^{2}$ every six weeks but, in the combination arm, myelosuppression at these doses required that lomustine be reduced to $90 \mathrm{mg} / \mathrm{m}^{2}$. These data have already influenced clinical practice and have served as the foundation for a phase III trial recently completed by the European Organization for the Treatment and Research of Cancer (EORTC-26101) comparing bevacizumab only with lomustine only (with crossover at progression) with the combination therapy. Results of this trial are expected to define, in a definitive way, the place of bevacizumab in the management of recurrent glioblastoma.

Assessment of radiographic response is problematic when antiangiogenic agents are being evaluated because drugs such as bevacizumab normalize tumour vasculature and decrease vascular permeability causing apparent improvement in the appearance of T1 post-gadolinium and FLAIR sequences on MR scans and these changes are typically interpreted as responses. Acknowledgement of this challenge has stimulated the development of the Response Assessment in Neuro-Oncology (RANO) criteria, a tool that attempts to account for the impact of antiangiogenic agents on radiographic and clinical assessment. ${ }^{21}$ Despite the difficulties in response assessment when using antiangiogenic agents, there are data to suggest that radiographic response has significance. ${ }^{22}$ Retrospective analysis of the BRAIN phase II trial evaluating the efficacy of bevacizumab alone and in combination with irinotecan identified objective radiographic response at 9, 18 and 26 weeks 
as a statistically significant predictor of survival. ${ }^{22}$ Additionally, progression-free survival at these time points was also a statistically significant predictor of survival.

The radiographic pattern of relapse for patients receiving bevacizumab is variable and, in a review of patients treated with bevacizumab for recurrent glioblastoma at Memorial SloanKettering Cancer Centre, $46 \%$ had increasing enhancement at the initial site of disease, $16 \%$ had new enhancement remote from the initial site of disease and $35 \%$ had predominantly progression of nonenhancing tumor. ${ }^{23}$ Progression of nonenhancing tumour is best appreciated on FLAIR sequences and the significance of this kind of tumour growth has generated much speculation. Nonenhancing progression represents an infiltrative pattern of recurrence and this has been interpreted by some as evidence that bevacizumab induces a more invasive phenotype, possibly accounting for the minimal impact this agent has on overall survival despite very impressive response rates and progressionfree survival. ${ }^{24}$ However, recent analyses of patterns of recurrence and survival in patients enrolled in the BRAIN and AVAglio (a randomized phase III trial of bevacizumab with radiotherapy and temozolomide in in patients with newly diagnosed glioblastoma) trials have noted that bevacizumab treatment did not cause more infiltrative disease at progression for both newly diagnosed and recurrent patients and that that survival in not influenced by whether recurrence is predominantly enhancing or not. $^{25,26}$

At progression on bevacizumab monotherapy, particularly when nonenhancing tumour growth is the predominant radiographic pattern, bevacizumab is often continued with either the addition of or change in chemotherapy. This behavior is common partly because nonenhancing progression is vague and hard to quantitate and is associated frequently with subtle clinical changes but also because of fears surrounding rapid clinical deterioration once bevacizumab is discontinued. ${ }^{27}$ There are some data to support this practice as suggested by a study that demonstrated a modest two-month prolongation in survival when bevacizumab was continued after documented failure of a bevacizumab-containing regimen. ${ }^{28}$ This retrospective study identified bevacizumab continuation as an independent predictor of improved overall survival $(p=0.04)$. The effectiveness of bevacizumab continuation beyond multiple progressions is being explored by an ongoing Roche sponsored trial in newly diagnosed glioblastoma (TAMIGA trial) and the results of this study should furnish important data on the usefulness of this approach. $^{29}$

When first approved in the United States, bevacizumab quickly became the drug of choice for recurrent glioblastoma at first progression. However, concerns surrounding toxicities, challenges of re-operation at the time of bevacizumab failure, fears surrounding the potential of bevacizumab to induce a more aggressive invasive phenotype and the absence of effective salvage therapies following bevacizumab failure have shifted use to later progressions. Additionally, at academic centres, bevacizumab initiation is often delayed to facilitate enrolment in clinical trials as many studies exclude patients who have had bevacizumab exposure because of concerns surrounding accurate radiographic response assessment and unpredictable clinical deterioration after antiangiogenic therapy is discontinued. The safety and efficacy of delaying use of bevacizumab for recurrent glioblastoma has been examined in a retrospective study of 468 patients treated with bevacizumab at different recurrences. ${ }^{30}$
This study reported no differences in progression-free survival (median, 4.1 months) and overall survival (median, 9.8 months) when bevacizumab is used at first, second or third and subsequent recurrences. These results suggest that for most patients with progressive glioblastoma, delaying bevacizumab does not reduce its efficacy and that the magnitude of clinical benefit is not influenced by when bevacizumab is administered. Admittedly, this study was retrospective and influenced by decisions of treating physicians and selection bias. A decision to delay bevacizumab therapy requires careful patient selection and this study identified patients who were aged 60 and older and those with a low extent of tumour resection as those unable to tolerate a delay in bevacizumab initiation.

Conditional approval of bevacizumab for recurrent glioblastoma by the US Food and Drug Administration and Health Canada was granted with the expectation that bevacizumab would be evaluated in prospective randomized trials in newly diagnosed glioblastoma and that efficacy in terms of an overall survival advantage would be demonstrated. Two well-conducted, randomized, placebocontrolled trials have been completed, one by an academic cooperative group (RTOG-0825) and the other sponsored by Roche (AVAglio). ${ }^{31,32}$ Both trials had similar designs, incorporating bevacizumab into standard radiotherapy and temozolomide for newly diagnosed glioblastoma, with similar patient characteristics and co-primary endpoints of progression-free survival and overall survival. Both trials demonstrated a three-to-four month improvement in progression-free survival, no impact on overall survival and an acceptable safety and tolerability profile. No clear explanation exists for why an improvement in progression-free survival has not translated into an increase in overall survival. One explanation relates to dilution of the advantage of early bevacizumab by a cross over effect of patients in the placebo group receiving bevacizumab at recurrence. However, in the AVAglio trial, post-hoc statistical analyses have demonstrated no major impact of cross over at progression on overall survival. Another possible explanation is that bevacizumab had no effect on progression-free survival, that the apparent response rates and improved progression-free survival were spurious and related to the ability of bevacizumab to diminish tumour blood vessel permeability and contrast-enhancement on MR images. Nonetheless, an improved progression-free survival may be of value even without an effect on survival if clinical benefit can be demonstrated during progression-free survival. To this extent both RTOG-0825 and AVAglio trials included quality of life and neurocognitive evaluations as secondary endpoints, with surprisingly and as yet unresolved divergent results. The AVAglio trial demonstrated improved or stable quality of life and performance status during progression-free survival while the RTOG-0825 study reported a worsening quality of life and a decline in cognitive status during this time. Given the absence of an overall survival advantage and questionable clinical significance of an apparent improved progression-free survival, bevacizumab cannot be considered appropriate therapy for patients with newly diagnosed disease. However, there may be unique subpopulations that might benefit from early bevacizumab exposure. Post-hoc gene expression analysis of tumor samples of patients enrolled in the AVAglio trial has identified a subset of patients with novel gene clusters who appear to have prolonged overall survival when treated with bevacizumab as part of initial therapy. ${ }^{33}$ These intriguing results require validation 
prospectively and in other data sets but suggest that perhaps some patients benefit from early bevacizumab therapy. Additionally, there have been reports of bevacizumab being used to facilitate the completion of radiochemotherapy with temozolomide in patients with very poor performance status and large tumours. ${ }^{34}$ Furthermore, there may be a role for bevacizumab in the symptomatic management of patients who experience severe clinical deterioration due to pseudoprogression following completion of cranial irradiation.

In Canada, the use of bevacizumab for patients with recurrent glioblastoma is increasing and, because substantial clinical responses are possible, has expanded to include occasional treatment of patients with relatively poor performance status who might otherwise receive only palliative care. The use of bevacizumab in poor performance status patients to improve quality of life, reduce corticosteroid requirements and potentially diminish the cost of hospitalization requires additional clinical evaluation with pharmacoeconomic endpoints. Unlike temozolomide and other alkylator chemotherapies, bevacizumab is effective in patients with MGMT methylated and unmethylated tumours. ${ }^{31,35}$ For this latter group of poor prognosis patients with chemoresistant tumours, bevacizumab may be the most effective medical therapy for tumour progression. While it is unclear whether there is any benefit from administering bevacizumab with chemotherapy, there has been an increasing tendency to combine bevacizumab with lomustine chemotherapy. When combined therapy is used, bevacizumab is occasionally continued either alone or with a second chemotherapy at subsequent progression, although the duration of bevacizumab therapy at this stage is usually brief. As the optimal timing to initiate bevacizumab therapy for recurrent glioblastoma is unclear, there has been an increasing tendency to defer initiation of bevacizumab because therapeutic options following bevacizumab failure are very limited, further treatment usually ineffective and survival predictably short.

The results of the RTOG-0825 and AVAglio trials are disappointing and will not change the temozolomide radiochemotherapy standard of care for glioblastoma. However, glioblastoma remains an intractable disease where there is an unmet need for effective therapies. Many patients in Canada do not receive temozolomide at diagnosis, and for those who do, the impact of temzolomide on survival is modest, with a median overall survival of 15.8 months reported in a retrospective review of patients treated at a Canadian academic cancer centre. ${ }^{3}$ Bevacizumab is clearly the most important new agent for glioblastoma since the introduction of temozomide, but unlike this agent, the position of bevaciuzumab in the management of glioblastoma is in the recurrent setting, where its use is often associated with objective radiographic responses that translate into symptom improvement and prolongation of progression-free survival. ${ }^{36}$ Bevacizumab is now commonly used for this indication and future development of this agent should involve studies that refine its use in the management of recurrence and identify biomarkers predictive of benefit.

\section{Disclosures}

WPM is a consultant for Hoffmann La Roche Ltd. and is a member of the Steering Committees of the Roche sponsored AVAglio and TAMIGA phase III trials of bevacizumab for glioblastoma.
This publication was made possible with funding from Hoffmann-La Roche Limited. The contents are solely the responsibility of the author and do not necessarily represent the official views of Roche or any member of the Roche Group of companies.

\section{REFERENCES}

1. CBTRUS Statistical Report: Primary Brain and Central Nervous System Tumours Diagnosed in the United States in 2005-2009. Neuro-Oncology. 2012;14(Supplement 5):v1-49.

2. Stupp R, Mason WP, van den Bent MJ, et al. Radiotherapy plus concomitant and adjuvant temozolomide for glioblastoma. New Engl J Med. 2005;352:987-96.

3. Lwin Z, MacFadden D, Al-Zahrani A, et al. Glioblastoma management in the temozolomide era: have we improved outcome? J Neurooncol. 2013;115:303-10.

4. Hegi ME, Diserens AC, Gorlia T, et al. MGMT gene silencing and benefit from temozolomide in glioblastoma. New Engl J Med. 2005;352:997-1003.

5. Stupp R, Hegi ME, Mason WP, et al. Effects of radiotherapy with concomitant and adjuvant temozolomide versus radiotherapy alone on survival in glioblastoma in a randomised phase III study: 5-year analysis of the EORTC-NCIC trial. Lancet Oncology. 2009;10:459-66.

6. Wong ET, Hess KR, Gleason MJ, et al. Outcomes and prognostic factors in recurrent glioma patients enrolled onto phase II clinical trials. J Clin Oncol. 1999;17:2572-8.

7. Ballman KV, Buckner JC, Brown PD, et al. The relationship between six-month progression-free survival and 12-month overall survival end points for phase II trials in patients with glioblastoma multiforme. Neuro-Oncology. 2007;9:29-38.

8. Beal K, Abrey LE, Gutin PH. Antiangiogenic agents in the treatment of recurrent or newly diagnosed glioblastoma: Analysis of single-agent and combined modality approaches. Radiat Oncol. 2011;6:2-15.

9. McNamara MG, Mason WP. Antiangiogenic therapies in glioblastoma multiforme. Expert Rev Anticancer Ther. 2012;12: 643-54.

10. Cohen MH, Shen YL, Keegan P, Pazdur R. FDA drug approval summary: bevacizumab (Avastin) as treatment of recurrent glioblastoma multiforme. Oncologist. 2009;14:1131-8.

11. Friedman HS, Prados MD, Wen PY, et al. Bevacizumab alone and in combination with irinotecan in recurrent glioblastoma. J Clin Oncol. 2009;27:4733-40.

12. Kreisl TN, Kim L, Moore K, et al. Phase II trial of single-agent bevacizumab followed by bevacizumab plus irinotecan at tumor progression in recurrent glioblastoma. J Clin Oncol. 2009;27: 740-5.

13. Wefel JS, Cloughesy T, Zazzali JL, et al. Neurocognitive function in patients with recurrent glioblastoma treated with bevacizumab. Neuro-Oncology. 2011;13:660-8.

14. Vredenburgh JJ, Cloughesy T, Samant M, et al. Corticosteroid use in patients with glioblastoma at first or second relapse treated with bevacizumab in the BRAIN study. Oncologist. 2010;15:1329-34.

15. Johnson DR, Leeper HE, Uhm JH. Glioblastoma survival in the United States improved after Food and Drug Administration approval of bevacizumab: a population-based analysis. Cancer. 2013;119:3489-95.

16. Galanis E, Anderson SK, Lafky JM, et al. Phase II study of bevacizumab in combination with sorafenib in recurrent glioblastoma (N0776): a north central cancer treatment group trial. Clin Cancer Res. 2013;19:4816-23.

17. Reardon DA, Desjardins A, Peters K, et al. Phase II study of metronomic chemotherapy with bevacizumab for recurrent glioblastoma after progression on bevacizumab therapy. J Neurooncol. 2011;103:371-9.

18. Lassen U, Sorensen M, Gaziel TB, Hasselbalch B, Poulsen HS. Phase II study of bevacizumab and temsirolimus combination therapy for recurrent glioblastoma multiforme. Anticancer Res. 2013;33:1657-60. 
19. Field KM, Simes J, Wheeler H, et al. A randomized phase II study of carboplatin and bevacizumab in recurrent glioblastoma multiforme (CABARET). J Clin Oncol. 2013;31 (suppl; abstract 2017).

20. Taal W, Oosterkamp HM, Walenkamp AME, et al. A randomized phase II study of bevacizumab versus bevacizumab plus lomustine versus lomustine single agent in recurrent glioblastoma: The Dutch BELOB study. J Clin Oncol. 2013;31 (suppl; abstract 2001).

21. Wen PY, Macdonald DR, Reardon DA, et al. Updated response assessment criteria for high-grade gliomas: response assessment in neuro-oncology working group. J Clin Oncol. 2010;28: 1963-72.

22. Prados M, Cloughesy T, Samant M, et al. Response as a predictor of survival in patients with recurrent glioblastoma treated with bevacizumab. Neuro-Oncology. 2011;13:143-51.

23. Iwamoto FM, Abrey LE, Beal K, et al. Patterns of relapse and prognosis after bevacizumab failure in recurrent glioblastoma. Neurology. 2009;73:1200-6.

24. Wen PY, Junck L. Bevacizumab for glioblastoma: what can we learn from patterns of progression? Neurology. 2014;82:1670-1.

25. Pope WB, Xia Q, Paton VE, et al. Patterns of progression in patients with recurrent glioblastoma treated with bevacizumab. Neurology. 2011;76:432-7.

26. Wick W, Chinot OL, Mason WP, et al. Patterns of tumour progression in a phase 3 study of bevacizumab (Bv) plus radiotherapy (RT) plus temozolomide (T) for newly diagnosed glioblastoma (GB). J Clin Oncol. 2014;32 (No. 15_suppl; abstract 2051).

27. Kesselheim JC, Norden AD, Wen PY, Joffe S. Discontinuing bevacizumab in patients with glioblastoma: an ethical analysis. Oncologist. 2011;16:1435-9.
28. Reardon DA, Herndon JE, 2nd, Peters KB, et al. Bevacizumab continuation beyond initial bevacizumab progression among recurrent glioblastoma patients. Brit J Cancer. 2012;107:1481-7.

29. Brandes AA, Mason W, Pichler J, et al. Clinical trial protocol: Can bevacizumab prolong survival for glioblastoma patients through multiple lines of therapy? Future Oncol. 2014;10:1137-45.

30. Piccioni DE, Selfridge J, Mody RR, et al. Deferred use of bevacizumab for recurrent glioblastoma is not associated with diminished efficacy. Neuro-Oncology. 2014;16:815-22.

31. Chinot OL, Wick W, Mason W, et al. Bevacizumab plus radiotherapy-temozolomide for newly diagnosed glioblastoma. New Engl J Med. 2014;370:709-22.

32. Gilbert MR, Dignam JJ, Armstrong TS, et al. A randomized trial of bevacizumab for newly diagnosed glioblastoma. New Engl J Med. 2014;370:699-708.

33. Phillips H, Sandmann T, Li C, et al. Correlation of molecular subtypes with survival in AVAglio (bevacizumab [Bv] and radiotherapy $[\mathrm{RT}]$ and temozolomide $[\mathrm{T}]$ for newly diagnosed glioblastoma [GB . J Clin Oncol. 2014;32 (No. 15_suppl; abstract 2001).

34. Green RM, Woyshner EA, Nghiemphu L, Lai A, Cloughesy T. Use of bevacizumab to facilitate up-front chemoradiation in poor-risk patients with glioblastoma multiforme by improving neurologic function. J Clin Oncol. 2010;20 (No. 15_suppl; abstract 2059).

35. Herrlinger U, Schaefer N, Steinbach JP, et al. Bevacizumab, irinotecan, and radiotherapy versus standard temozolomide and radiotherapy in newly diagnosed, MGMT-nonmethylated glioblastoma patients: First results from the randomized multicenter GLARIUS trial. J Clin Oncol. 2013;31 (suppl; abstract LBA2000).

36. Fine HA. Bevacizumab in glioblastoma-still much to learn. New Engl J Med. 2014;370:764-5. 